\title{
PROOF OF THE ARNOLD CHORD CONJECTURE IN THREE DIMENSIONS I
}

\author{
Michael Hutchings and Clifford Henry Taubes
}

\begin{abstract}
This paper and its sequel prove that every Legendrian knot in a closed threemanifold with a contact form has a Reeb chord. The present paper deduces this result from another theorem, asserting that an exact symplectic cobordism between contact 3-manifolds induces a map on (filtered) embedded contact homology satisfying certain axioms. The latter theorem will be proved in the sequel using Seiberg-Witten theory.
\end{abstract}

\section{Introduction}

1.1. The chord conjecture. Let $Y$ be a closed oriented 3-manifold (all 3-manifolds in this paper will be assumed connected). Recall that a contact form on $Y$ is a 1form $\lambda$ on $Y$ with $\lambda \wedge d \lambda>0$ everywhere. The contact form $\lambda$ determines a contact structure, namely the oriented 2-plane field $\xi=\operatorname{Ker}(\lambda)$. It also determines the Reeb vector field $R$ characterized by $d \lambda(R, \cdot)=0$ and $\lambda(R)=1$. A Legendrian knot in $(Y, \lambda)$ is a smooth knot $\mathcal{K} \subset Y$ such that $\left.T \mathcal{K} \subset \xi\right|_{\mathcal{K}}$. A Reeb chord of $\mathcal{K}$ is a trajectory of the Reeb vector field starting and ending on $\mathcal{K}$, i.e. a path $\gamma:[0, T] \rightarrow Y$ for some $T>0$ such that $\gamma^{\prime}(t)=R(\gamma(t))$ and $\gamma(0), \gamma(T) \in \mathcal{K}$. The main result of this paper is:

Theorem 1.1. Let $Y$ be a closed oriented 3-manifold with a contact form $\lambda$. Then every Legendrian knot in $(Y, \lambda)$ has a Reeb chord.

This is a version of a conjecture of Arnold [2]. For the 3-sphere with any contact form inducing the standard contact structure, and more generally for boundaries of subcritical Stein manifolds in any odd dimension, this was proved by Mohnke [11]. This was also proved by Abbas [1] for Legendrian unknots in tight contact 3-manifolds satisfying certain assumptions.

The proof of Theorem 1.1 given here uses the relationship between embedded contact homology and Seiberg-Witten Floer cohomology. We now recall the relevant parts of this correspondence.

1.2. Embedded contact homology. We begin by briefly reviewing the definition of embedded contact homology. For more details see [6] and the references therein.

Let $Y$ be a closed oriented 3-manifold with a contact form $\lambda$. A Reeb orbit is a closed orbit of the Reeb vector field, i.e. a map $\gamma: \mathbb{R} / T \mathbb{Z} \rightarrow Y$ for some $T>0$ with $\gamma^{\prime}(t)=R(\gamma(t))$, modulo reparametrization. The linearized Reeb flow along a Reeb orbit $\gamma$ defines an endomorphism $P_{\gamma}$ of the 2-dimensional symplectic vector space $\left(\xi_{\gamma(0)}, d \lambda\right)$. A Reeb orbit $\gamma$ is nondegenerate if $P_{\gamma}$ does not have 1 as an eigenvalue. In this case either $P_{\gamma}$ has real eigenvalues, in which case we say that $\gamma$ is hyperbolic,

Received by the editors June 9, 2010. 
or $P_{\gamma}$ has eigenvalues on the unit circle, in which case $\gamma$ is called elliptic. These notions do not depend on the parametrization of $\gamma$. We say that the contact form $\lambda$ is nondegenerate if all its Reeb orbits are nondegenerate. A generic contact form has this property.

Assume now that the contact form $\lambda$ on $Y$ is nondegenerate. An orbit set is a finite set of pairs $\Theta=\left\{\left(\Theta_{i}, m_{i}\right)\right\}$ where the $\Theta_{i}$ 's are distinct embedded Reeb orbits, and the $m_{i}$ 's are positive integers which one can think of as "multiplicities". The homology class of the orbit set $\Theta$ is defined by

$$
[\Theta]:=\sum_{i} m_{i}\left[\Theta_{i}\right] \in H_{1}(Y) .
$$

The orbit set $\Theta=\left\{\left(\Theta_{i}, m_{i}\right)\right\}$ is called admissible if $m_{i}=1$ whenever $\Theta_{i}$ is hyperbolic. An admissible orbit set is also called an ECH generator.

If $\Gamma \in H_{1}(Y)$, then the embedded contact homology $E C H_{*}(Y, \lambda, \Gamma)$ is the homology of a chain complex which is freely generated over $\mathbb{Z} / 2$ by admissible orbit sets $\Theta$ with $[\Theta]=\Gamma$.

Convention. Although ECH is ordinarily defined over $\mathbb{Z}$, with the signs specified in $[9, \S 9]$, in this paper ECH is always defined with $\mathbb{Z} / 2$ coefficients, because this is sufficient for the applications here and will allow us to avoid orientation headaches.

To define the chain complex differential one chooses a generic almost complex structure $J$ on $\mathbb{R} \times Y$ of the following type:

Definition 1.2. An almost complex structure $J$ on $\mathbb{R} \times Y$ is symplectization-admissible if $J$ is $\mathbb{R}$-invariant, $J\left(\partial_{s}\right)=R$ where $s$ denotes the $\mathbb{R}$ coordinate, and $J$ sends $\xi$ to itself, rotating $\xi$ positively with respect to the orientation on $\xi$ given by $d \lambda$.

The reason for the terminology is that the noncompact symplectic manifold ( $\mathbb{R} \times$ $\left.Y, d\left(e^{s} \lambda\right)\right)$ is called the symplectization of $(Y, \lambda)$.

Given a symplectization-admissible $J$, we now consider (not necessarily embedded) $J$-holomorphic curves in $\mathbb{R} \times Y$ whose domains are (not necessarily connected) punctured compact Riemann surfaces. A positive end of such a holomorphic curve at a (not necessarily embedded) Reeb orbit $\gamma$ is an end which is asymptotic to the cylinder $\mathbb{R} \times \gamma$ as the $\mathbb{R}$ coordinate $s \rightarrow+\infty$. A negative end is defined analogously with $s \rightarrow-\infty$.

Definition 1.3. Given a symplectization-admissible $J$, and given (not necessarily admissible) orbit sets $\Theta=\left\{\left(\Theta_{i}, m_{i}\right)\right\}$ and $\Theta^{\prime}=\left\{\left(\Theta_{j}^{\prime}, m_{j}^{\prime}\right)\right\}$, a " $J$-holomorphic curve from $\Theta$ to $\Theta^{\prime \prime \prime}$ is a $J$-holomorphic curve in $\mathbb{R} \times Y$ as above with positive ends at covers of $\Theta_{i}$ with total multiplicity $m_{i}$, negative ends at covers of $\Theta_{j}^{\prime}$ with total multiplicity $m_{j}^{\prime}$, and no other ends.

Such a holomorphic curve may be multiply covered, but we are only interested in the corresponding current. In particular, let $\mathcal{M}^{J}\left(\Theta, \Theta^{\prime}\right)$ denote the moduli space of $J$-holomorphic curves from $\Theta$ to $\Theta^{\prime}$, where two such curves are considered equivalent if they represent the same current in $\mathbb{R} \times Y$, up to translation of the $\mathbb{R}$ coordinate.

Given ECH generators $\Theta$ and $\Theta^{\prime}$ with $[\Theta]=\left[\Theta^{\prime}\right]=\Gamma$, the differential coefficient $\left\langle\partial \Theta, \Theta^{\prime}\right\rangle \in \mathbb{Z} / 2$ is the $\bmod 2$ count of $J$-holomorphic curves in $\mathcal{M}^{J}\left(\Theta, \Theta^{\prime}\right)$ with "ECH index" equal to 1 . The definition of the ECH index is not needed in this paper 
and may be found in [5]. If $J$ is generic, then $\partial$ is well-defined and $\partial^{2}=0$, as shown in $[8, \S 7]$. In this case we denote the chain complex by $E C C_{*}(Y, \lambda, \Gamma ; J)$. A symplectization-admissible almost complex structure that is generic in this sense will be called ECH-generic here. It turns out that the curves counted by the ECH differential are embedded, except that they may include multiple covers of $\mathbb{R}$-invariant cylinders. The ECH index defines a relative $\mathbb{Z} / d\left(c_{1}(\xi)+2 \mathrm{PD}(\Gamma)\right)$ grading on the chain complex, where $d$ denotes divisibility in $H^{2}(Y ; \mathbb{Z}) /$ Torsion.

1.3. The isomorphism with Seiberg-Witten Floer cohomology. Although the ECH differential depends on the choice of $J$, the homology of the chain complex does not. This follows from a much stronger theorem of the second author $[15,16,17,18]$ asserting that ECH is isomorphic to a version of Seiberg-Witten Floer cohomology as defined by Kronheimer-Mrowka. To be precise, there are three basic versions of Seiberg-Witten Floer cohomology, denoted by $\widehat{H M}^{*}, \widehat{H M}^{*}$, and $\overline{H M}^{*}$. The first of these is the one that is relevant to ECH; it assigns $\mathbb{Z} / 2$-modules $\widehat{H M}^{*}(Y, \mathfrak{s})$ to each spin-c structure $\mathfrak{s}$ on $Y$, which have a relative $\mathbb{Z} / d\left(c_{1}(\mathfrak{s})\right)$-grading.

Convention 1.4. In this paper, all Seiberg-Witten Floer cohomology is defined with $\mathbb{Z} / 2$ coefficients (even though it can be defined over $\mathbb{Z}$, which is the default coefficient system in [12]).

Recall that the set $\operatorname{Spin}^{c}(Y)$ of spin-c structures on $Y$ is an affine space over $H^{2}(Y ; \mathbb{Z})$, and the contact structure $\xi$ determines a distinguished spin-c structure $\mathfrak{s}_{\xi}$. With this convention, the theorem is now that

$$
E C H_{*}(Y, \lambda, \Gamma) \simeq \widehat{H M}^{-*}\left(Y, \mathfrak{s}_{\xi}+\operatorname{PD}(\Gamma)\right),
$$

as relatively graded $\mathbb{Z} / 2$-modules. (There is also an isomorphism with $\mathbb{Z}$ coefficients [17].)

It follows from scrutiny of the proof of (1.1), together with the invariance properties of $\widehat{H M}^{*}$, that the versions of $E C H_{*}(Y, \lambda, \Gamma)$ defined using different almost complex structures $J$ are canonically isomorphic to each other. This point is explained in detail in [10]. Thus it makes sense to talk about $E C H_{*}(Y, \lambda, \Gamma)$ without referring to a choice of $J$. Moreover, under this identification, the isomorphism (1.1) is canonical.

At times it is convenient to ignore the homology class $\Gamma$ in the definition of $E C H$, and simply define

$$
E C H_{*}(Y, \lambda):=\bigoplus_{\Gamma \in H_{1}(Y)} E C H_{*}(Y, \lambda, \Gamma) .
$$

This is the homology of a chain complex $E C C_{*}(Y, \lambda ; J)$ generated by all admissible orbit sets, and by (1.1) this homology is canonically isomorphic (as a relatively graded $\mathbb{Z} / 2$-module) to

$$
\widehat{H M}^{-*}(Y):=\bigoplus_{\mathfrak{s} \in \operatorname{Spin}^{c}(Y)} \widehat{H M}^{-*}(Y, \mathfrak{s})
$$

The proof of Theorem 1.1 makes use of two additional structures on ECH: the action filtration and cobordism maps. We now explain these. 
1.4. The action filtration. If $\Theta=\left\{\left(\Theta_{i}, m_{i}\right)\right\}$ is an orbit set, its symplectic action or length is defined by

$$
\mathcal{A}(\Theta):=\sum_{i} m_{i} \int_{\Theta_{i}} \gamma
$$

The ECH differential for any (generic) symplectization-admissible $J$ decreases the action, i.e. if $\left\langle\partial \Theta, \Theta^{\prime}\right\rangle \neq 0$ then $\mathcal{A}(\Theta) \geq \mathcal{A}\left(\Theta^{\prime}\right)$. This is because if $C \in \mathcal{M}^{J}\left(\Theta, \Theta^{\prime}\right)$, then $\left.d \lambda\right|_{C} \geq 0$ everywhere $^{1}$. Thus for any real number $L$, it makes sense to define the filtered $E C H$, denoted by $E C H_{*}^{L}(Y, \lambda)$, to be the homology of the subcomplex $E C C_{*}^{L}(Y, \lambda ; J)$ of the ECH chain complex spanned by ECH generators with action less than $L$. It is shown in [10] that $E C H_{*}^{L}(Y, \lambda)$, just like $E C H_{*}(Y, \lambda)$, does not depend on the choice of ECH-generic $J$. However $\operatorname{ECH}_{*}^{L}(Y, \lambda)$, unlike the usual ECH, can change when one deforms the contact form $\lambda$.

For $L<L^{\prime}$ there is a map

$$
\imath^{L, L^{\prime}}: E C H_{*}^{L}(Y, \lambda) \longrightarrow E C H_{*}^{L^{\prime}}(Y, \lambda)
$$

induced by the inclusion of chain complexes (for some given $J$, although it is shown in [10] that (1.3) does not depend on $J$ ). The usual ECH is recovered as the direct limit

$$
E C H_{*}(Y, \lambda)=\lim _{L \rightarrow \infty} E C H_{*}^{L}(Y, \lambda)
$$

In particular, there is a natural map

$$
\imath^{L}: E C H_{*}^{L}(Y, \lambda) \longrightarrow E C H_{*}(Y, \lambda),
$$

again induced by an inclusion of chain complexes.

1.5. Cobordism maps in ECH. Let $\left(Y_{+}, \lambda_{+}\right)$and $\left(Y_{-}, \lambda_{-}\right)$be closed oriented 3manifolds with nondegenerate contact forms. An exact symplectic cobordism from $\left(Y_{+}, \lambda_{+}\right)$to $\left(Y_{-}, \lambda_{-}\right)$is a compact symplectic 4-manifold $(X, \omega)$ with boundary $\partial X=$ $Y_{+}-Y_{-}$, for which there exists a 1 -form $\lambda$ on $X$ such that $d \lambda=\omega$ on $X$ and $\left.\lambda\right|_{Y_{ \pm}}=\lambda_{ \pm}$. A 1-form $\lambda$ as above is called a Liouville form for $(X, \omega)$. When we wish to specify a Liouville form (which we usually do), we denote the exact symplectic cobordism by $(X, \lambda)$, and we continue to write $\omega=d \lambda$.

Note that our designation of the cobordism as "from $Y_{+}$to $Y_{-}$" is natural from the perspective of symplectic geometry, but opposite from the usual convention in Seiberg-Witten and Heegaard Floer homology. This is connected with the fact that embedded contact homology is identified with Seiberg-Witten Floer cohomology.

\footnotetext{
${ }^{1}$ In fact if $\left\langle\partial \Theta, \Theta^{\prime}\right\rangle \neq 0$ then the strict inequality $\mathcal{A}(\Theta)>\mathcal{A}\left(\Theta^{\prime}\right)$ holds, because $d \lambda$ vanishes identically on $C$ if and only if the image of $C$ is $\mathbb{R}$-invariant, in which case $C$ has ECH index zero and cannot contribute to the differential.
} 
Now let $(X, \lambda)$ be an exact symplectic cobordism as above. This cobordism, like any smooth cobordism, induces a map ${ }^{2}$ of ungraded $\mathbb{Z} / 2$-modules from the SeibergWitten Floer cohomology of $Y_{+}$to that of $Y_{-}$, which we denote by

$$
\widehat{H M}^{*}(X): \widehat{H M}^{*}\left(Y_{+}\right) \longrightarrow \widehat{H M}^{*}\left(Y_{-}\right) \text {. }
$$

Definition 1.5. Define

$$
\Phi(X): E C H_{*}\left(Y_{+}, \lambda_{+}\right) \longrightarrow E C H_{*}\left(Y_{-}, \lambda_{-}\right)
$$

to be the map on ECH obtained by composing the map (1.6) on Seiberg-Witten Floer cohomology with the canonical isomorphism (1.1) on both sides.

It is natural to expect that the map (1.7) can be defined directly, without using Seiberg-Witten theory, by suitably counting holomorphic curves in the "completion" of $(X, \lambda)$. The latter is a noncompact symplectic manifold defined as follows. To start, one can find $\varepsilon>0$, a neighborhood $N_{-}$of $Y_{-}$in $X$, and an identification $N_{-} \simeq[0, \varepsilon) \times Y_{-}$, such that $\lambda=e^{s} \lambda_{-}$on $N_{-}$, where $s$ denotes the $[0, \varepsilon)$ coordinate. The requisite map $[0, \varepsilon) \times Y_{-} \rightarrow X$ is obtained using the flow starting at $Y_{-}$of the unique vector field $V$ on $X$ such that $\imath_{V} \omega=\lambda$. Likewise, a neighborhood $N_{+}$of $Y_{+}$in $X$ can be identified with $(-\varepsilon, 0] \times Y_{+}$so that $\lambda=e^{s} \lambda_{+}$on $N_{+}$. Using these identifications, one can then glue symplectization ends to $X$ to obtain the completion

$$
\bar{X}:=\left((-\infty, 0] \times Y_{-}\right) \cup_{Y_{-}} X \cup_{Y_{+}}\left([0, \infty) \times Y_{+}\right) .
$$

Note for reference later that the Liouville form $\lambda$ on $X$ canonically extends to a 1-form on $\bar{X}$ which equals $e^{s} \lambda_{ \pm}$on the ends.

Definition 1.6. An almost complex structure $J$ on $\bar{X}$ is cobordism-admissible if it is $\omega$-compatible ${ }^{3}$ on $X$, and if it agrees with symplectization-admissible almost complex structures $J_{+}$for $\lambda_{+}$on $[0, \infty) \times Y_{+}$and $J_{-}$for $\lambda_{-}$on $(-\infty, 0] \times Y_{-}$.

Given a cobordism-admissible $J$, and given (not necessarily admissible) orbit sets $\Theta^{+}=\left\{\left(\Theta_{i}^{+}, m_{i}^{+}\right)\right\}$in $Y_{+}$and $\Theta^{-}=\left\{\left(\Theta_{j}^{-}, m_{i}^{-}\right)\right\}$in $Y_{-}$, we define a " $J$-holomorphic curve in $\bar{X}$ from $\Theta^{+}$to $\Theta^{-}$" analogously to Definition 1.3, and denote the moduli space of such curves by $\mathcal{M}^{J}\left(\Theta^{+}, \Theta^{-}\right)$, where two such curves are considered equivalent if they represent the same current in $\bar{X}$.

One would now like to define the map (1.7) by choosing a generic cobordismadmissible $J$ and suitably counting $J$-holomorphic curves in $\bar{X}$ as above with ECH index 0 , so as to define a chain map between the ECH chain complexes which induces the map (1.7) on homology. (In general one also needs to include contributions from

\footnotetext{
${ }^{2}$ Kronheimer-Mrowka define this map on the "completed" Seiberg-Witten Floer cohomology $\widehat{H M}^{\bullet}$. However for $\widehat{H M}$, the completed and uncompleted cohomologies are the same. Completion only makes a difference for the alternate versions $\overline{H M}^{*}$ and $\overline{H M}^{*}$ of Seiberg-Witten Floer cohomology.

Note also that if one uses coefficients in $\mathbb{Z}$ instead of $\mathbb{Z} / 2$, then the signs in the cobordism map on $\widehat{H M}$ depend on a choice of "homology orientation" of $X$. However one expects to be able to define cobordism maps on $E C H$ over $\mathbb{Z}$ without making such a choice, cf. [14, Lem. A.14]. Presumably an exact symplectic cobordism has a canonical homology orientation which makes the signs agree.

${ }^{3}$ Everything we describe below should still be possible if one weakens the $\omega$-compatible condition here to $\omega$-tame. However, because the papers relating Seiberg-Witten Floer cohomology to ECH use compatible almost complex structures, we will stick with the latter to avoid confusion.
} 
"broken" J-holomorphic curves, see §2.) An important consequence of such a construction would be that the map (1.7) respects the action filtrations, i.e. is induced by a chain map which does not increase the action filtration. The reason is that if $C$ is any holomorphic curve in $\mathcal{M}^{J}\left(\Theta^{+}, \Theta^{-}\right)$, then by Stokes' theorem and the exactness of the cobordism we have

$$
\mathcal{A}\left(\Theta^{+}\right)-\mathcal{A}\left(\Theta^{-}\right)=\int_{C \cap[0, \infty) \times Y_{+}} d \lambda_{+}+\int_{C \cap X} \omega+\int_{C \cap(-\infty, 0] \times Y_{-}} d \lambda_{-},
$$

and all of the integrands on the right hand side are pointwise nonnegative by our assumptions on $J$.

Unfortunately it is not currently known how to define the map (1.7) in terms of holomorphic curves as above. The difficulty is that, as explained in [5, §5], the compactifications of the relevant moduli spaces of holomorphic curves can include broken curves with negative index multiply covered components, and it is not clear in general what these should contribute to the count (although examples show that such broken curves must sometimes make nonzero contributions). However we can still use Seiberg-Witten theory to show that the map (1.7) respects the action filtrations (in a slightly weaker sense than above), and enjoys some other useful properties which would follow from a definition in terms of holomorphic curves. The precise statement uses filtered ECH and is given in Theorem 2.4 below.

The basic idea of the proof of Theorem 2.4 is to perturb the Seiberg-Witten equations on $\bar{X}$ using a large multiple of the symplectic form, much as in the proof of (1.1), and to show that with such a perturbation, Seiberg-Witten solutions that contribute to the cobordism map (1.7) give rise to (possibly broken) holomorphic curves. The main analytical machinery is adapted from the proof of $(1.1)$ in $[15,18]$. Nonetheless the detailed proof is still long, so we have deferred it to the sequel [10].

1.6. Legendrian surgery. Returning finally to the chord conjecture, let $\left(Y_{0}, \lambda_{0}\right)$ be a closed oriented 3 -manifold with a contact form, and let $\mathcal{K}$ be a Legendrian knot in $\left(Y_{0}, \lambda_{0}\right)$. The contact structure determines a framing of $\mathcal{K}$, which we denote by $t b(\mathcal{K})$. Let $Y_{1}$ denote the 3 -manifold obtained by surgery on $\mathcal{K}$ with framing $t b(\mathcal{K})-1$. The surgery procedure determines a smooth cobordism $X$ from $Y_{1}$ to $Y_{0}$. As was shown in [20] and as we review in $\S 3$, the 3 -manifold $Y_{1}$ has a natural contact structure, which can be expressed as the kernel of a contact form $\lambda_{1}$ such that $X$ has the structure of an exact symplectic cobordism from $\left(Y_{1}, \lambda_{1}\right)$ to $\left(Y_{0}, \lambda_{0}\right)$. Moreover, as is familiar from the work of Bourgeois-Ekholm-Eliashberg [4] on Legendrian surgery in contact homology, the contact form $\lambda_{1}$ can be chosen so that, modulo "long" Reeb orbits, one has:

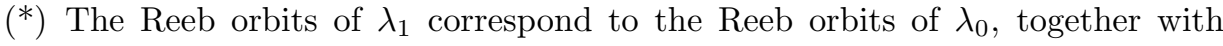
cyclic words in the Reeb chords of $\mathcal{K}$.

In particular, if $\mathcal{K}$ has no Reeb chord, then $\lambda_{1}$ and $\lambda_{0}$ have the same "short" Reeb orbits.

The idea of the proof of the chord conjecture is to use the preceding observation, together with Theorem 2.4 regarding the properties of ECH cobordism maps, to show that if there is no Reeb chord then the ECH cobordism map

$$
\Phi(X): E C H_{*}\left(Y_{1}, \lambda_{1}\right) \longrightarrow E C H_{*}\left(Y_{0}, \lambda_{0}\right)
$$


induced by the Legendrian surgery cobordism is an isomorphism. Note that this is what one would expect by analogy with a very special case of the aforementioned work of Bourgeois-Ekholm-Eliashberg.

But the map (1.10) cannot be an isomorphism, because this would contradict results of Kronheimer-Mrowka, namely:

Lemma 1.7. If $Y_{1}$ is obtained from a closed oriented 3-manifold $Y_{0}$ by surgery along a knot $\mathcal{K}$, and if $X$ denotes the corresponding smooth cobordism from $Y_{1}$ to $Y_{0}$, then the induced map on Seiberg-Witten Floer cohomology with $\mathbb{Z} / 2$ coefficients,

$$
\widehat{H M}^{*}(X): \widehat{H M}^{*}\left(Y_{1}\right) \longrightarrow \widehat{H M}^{*}\left(Y_{0}\right)
$$

is not an isomorphism.

Proof. It follows from [12, Thm. 42.2.1], see also [3, 13], that there is an exact triangle

$$
\cdots \longrightarrow \widehat{H M}^{*}\left(Y_{2}\right) \longrightarrow \widehat{H M}^{*}\left(Y_{1}\right) \stackrel{\widehat{H M}^{*}(X)}{\longrightarrow} \widehat{H M}^{*}\left(Y_{0}\right) \longrightarrow \widehat{H M}^{*}\left(Y_{2}\right) \longrightarrow \cdots
$$

where $Y_{2}$ is obtained from $Y_{0}$ by a certain different surgery along $\mathcal{K}$. Note that the exact triangle was only proved over $\mathbb{Z} / 2$, so it is fortunate that we are using $\mathbb{Z} / 2$ coefficients everywhere. Now the exact triangle implies that if (1.11) were an isomorphism, then $\widehat{H M}^{*}\left(Y_{2}\right)$ would vanish. However the latter is nontrivial, because it follows from [12, Cor. 35.1.4] that for any 3-manifold $Y$, if $\mathfrak{s}$ is a torsion spin-c structure on $Y$ (these always exist), then $\widehat{H M}^{*}(Y, \mathfrak{s})$ is infinitely generated. This is proved in [12] with $\mathbb{Z}$ coefficients, which immediately implies the statement with $\mathbb{Z} / 2$ coefficients.

There are two wrinkles in the above argument. First, statement $(*)$ is true only for Reeb orbits whose action is not too large, where the definition of "large" depends on the details of the Legendrian surgery construction. However one can modify $\lambda_{1}$ so as to make the corresponding upper bound on the action arbitrary large, see Lemma 3.2 below. Moreover the different versions of $\left(Y_{1}, \lambda_{1}\right)$ fit into a sequence of exact cobordisms. As a result, by making appropriate use of the cobordism maps on filtered $\mathrm{ECH}$, we can still show that if there is no Reeb chord then the ECH cobordism map (1.10) induced by the Legendrian surgery is an isomorphism.

Second, the above argument only makes sense if the contact form $\lambda_{0}$ (and with it the contact form $\lambda_{1}$ ) is nondegenerate, so that its ECH chain complex is well-defined. A priori there could exist a degenerate contact form and a Legendrian knot with no Reeb chord, such that for any nondegenerate perturbation of the contact form the knot does have a Reeb chord. To deal with this issue, we will show that when $\lambda_{0}$ is nondegenerate, there exists a Reeb chord with an upper bound on its symplectic action, given by a quantitative measure of the failure of the cobordism map (1.10) to be an isomorphism. The precise statement is given in Theorem 4.4 below. The aforementioned upper bound depends "continuously" on the contact form, as shown in Proposition 5.1. It then follows from a compactness argument that the chord conjecture holds in the degenerate case as well.

Contents of the rest of the paper. In $\S 2$ we give the precise statement of Theorem 2.4 on the existence and properties of maps on (filtered) ECH induced by exact symplectic cobordisms. In $\S 3-\S 5$ we use Theorem 2.4 as a "black box" to prove the 
chord conjecture. The formal proof of the chord conjecture is put together at the end of $\S 5$. In the sequel [10] we use Seiberg-Witten theory to prove Theorem 2.4.

\section{ECH and exact symplectic cobordisms}

We now state the theorem on the existence and properties of maps on (filtered) ECH induced by exact symplectic cobordisms.

We need some preliminary definitions. Below, let $(X, \lambda)$ be an exact symplectic cobordism from $\left(Y_{+}, \lambda_{+}\right)$to $\left(Y_{-}, \lambda_{-}\right)$, and assume that the contact forms $\lambda_{ \pm}$are nondegenerate. Fix a cobordism-admissible almost complex structure $J$ on $\bar{X}$ which restricts to symplectization-admissible almost complex structures $J_{+}$on $[0, \infty) \times Y_{+}$ and $J_{-}$on $(-\infty, 0] \times Y_{-}$, as in Definition 1.6.

Broken curves. Let $\Theta^{+}$and $\Theta^{-}$be (not necessarily admissible) orbit sets in $\left(Y_{+}, \lambda_{+}\right)$ and $\left(Y_{-}, \lambda_{-}\right)$respectively.

Definition 2.1. A broken J-holomorphic curve from $\Theta^{+}$to $\Theta^{-}$is a collection of holomorphic curves $\left\{C_{k}\right\}_{1 \leq k \leq N}$, and (not necessarily admissible) orbit sets $\Theta^{k+}$ and $\Theta^{k-}$ for each $k$, such that there exists $k_{0} \in\{1, \ldots, N\}$ such that:

- $\Theta^{k+}$ is an orbit set in $\left(Y_{+}, \lambda_{+}\right)$for each $k \geq k_{0} ; \Theta^{k-}$ is an orbit set in $\left(Y_{-}, \lambda_{-}\right)$ for each $k \leq k_{0} ; \Theta^{N+}=\Theta^{+} ; \Theta^{1-}=\Theta^{-}$; and $\Theta^{k-}=\Theta^{k-1,+}$ for each $k>1$.

- If $k>k_{0}$ then $C_{k} \in \mathcal{M}^{J_{+}}\left(\Theta^{k+}, \Theta^{k-}\right) ; C_{k_{0}} \in \mathcal{M}^{J}\left(\Theta^{k_{0},+}, \Theta^{k_{0},-}\right)$; and if $k<k_{0}$ then $C_{k} \in \mathcal{M}^{J_{-}}\left(\Theta^{k+}, \Theta^{k-}\right)$.

- If $k \neq k_{0}$ then $C_{k}$ is not $\mathbb{R}$-invariant (as a current).

Let $\overline{\mathcal{M}^{J}\left(\Theta^{+}, \Theta^{-}\right)}$denote the moduli space of broken $J$-holomorphic curves from $\Theta^{+}$ to $\Theta^{-}$as above.

Note that $\mathcal{M}^{J}\left(\Theta^{+}, \Theta^{-}\right)$is a subset of $\overline{\mathcal{M}^{J}\left(\Theta^{+}, \Theta^{-}\right)}$corresponding to broken curves as above in which $N=1$ (and it is perhaps a misnomer to call such curves "broken").

Product cylinders. If the cobordism $(X, \lambda)$ and the almost complex structure $J$ on $\bar{X}$ are very special, then $X$ may contain regions that look like pieces of a symplectization, in the following sense:

Definition 2.2. A product region in $X$ is the image of an embedding $\left[s_{-}, s_{+}\right] \times Z \rightarrow$ $X$, where $s_{-}<s_{+}$and $Z$ is an open 3-manifold, such that:

- $\left\{s_{ \pm}\right\} \times Z$ maps to $Y_{ \pm}$, and $\left(s_{-}, s_{+}\right) \times Z$ maps to the interior of $X$.

- The pullback of the Liouville form $\lambda$ to $\left[s_{-}, s_{+}\right] \times Z$ has the form $e^{s} \lambda_{0}$, where $s$ denotes the $\left[s_{-}, s_{+}\right]$coordinate, and $\lambda_{0}$ is a contact form on $Z$.

- The pullback of the almost complex structure $J$ to $\left[s_{-}, s_{+}\right] \times Z$ has the following properties:

- The restriction of $J$ to $\operatorname{Ker}\left(\lambda_{0}\right)$ is independent of $s$.

- $J(\partial / \partial s)=f(s) R_{0}$, where $f$ is a positive function of $s$ and $R_{0}$ denotes the Reeb vector field for $\lambda_{0}$.

Given a product region as above, the embedded Reeb orbits of $\lambda_{ \pm}$in $\left\{s_{ \pm}\right\} \times Z$ are identified with the embedded Reeb orbits of $\lambda_{0}$ in $Z$. If $\gamma$ is such a Reeb orbit, then we can form a $J$-holomorphic cylinder in $\bar{X}$ by taking the union of $\left[s_{-}, s_{+}\right] \times \gamma$ in $\left[s_{-}, s_{+}\right] \times Z$ with $(-\infty, 0] \times \gamma$ in $(-\infty, 0] \times Y_{-}$and $[0, \infty) \times \gamma$ in $[0, \infty) \times Y_{+}$.

Definition 2.3. We call a $J$-holomorphic cylinder as above a product cylinder. 
Composition of cobordisms. If $\left(X_{1}, \lambda_{1}\right)$ is an exact symplectic cobordism from $\left(Y_{+}, \lambda_{+}\right)$to $\left(Y_{0}, \lambda_{0}\right)$, and if $\left(X_{2}, \lambda_{2}\right)$ is an exact symplectic cobordism from $\left(Y_{0}, \lambda_{0}\right)$ to $\left(Y_{-}, \lambda_{-}\right)$, then we can compose them to obtain an exact symplectic cobordism $\left(X_{2} \circ X_{1}, \lambda\right)$ from $\left(Y_{+}, \lambda_{+}\right)$to $\left(Y_{-}, \lambda_{-}\right)$. Here $X_{2} \circ X_{1}$ is obtained by gluing $X_{1}$ and $X_{2}$ along $Y_{0}$ analogously to (1.8), and $\left.\lambda\right|_{X_{i}}=\lambda_{i}$ for $i=1,2$.

Homotopy of cobordisms. Two exact symplectic cobordisms $\left(X, \omega_{0}\right)$ and $\left(X, \omega_{1}\right)$ from $\left(Y_{+}, \lambda_{+}\right)$to $\left(Y_{-}, \lambda_{-}\right)$with the same underlying four-manifold $X$ are homotopic if there is a one-parameter family of symplectic forms $\left\{\omega_{t} \mid t \in[0,1]\right\}$ on $X$ such that $\left(X, \omega_{t}\right)$ is an exact symplectic cobordism from $\left(Y_{+}, \lambda_{+}\right)$to $\left(Y_{-}, \lambda_{-}\right)$for each $t \in[0,1]$.

Scaling. If $\lambda$ is a nondegenerate contact form on $Y$, and if $c$ is a positive constant, then there is a canonical "scaling" isomorphism

$$
s: \operatorname{ECH}_{*}^{L}(Y, \lambda) \stackrel{\simeq}{\longrightarrow} E C H_{*}^{c L}(Y, c \lambda) .
$$

To see this, observe that the chain complexes on both sides have the same generators. Moreover, an ECH-generic almost complex structure $J$ for $\lambda$ induces a symplectization-admissible almost complex structure $J^{c}$ for $c \lambda$, such that $J$ and $J^{c}$ agree when restricted to the contact planes $\xi$. The self-diffeomorphism of $\mathbb{R} \times Y$ sending $(s, y) \mapsto(c s, y)$ then induces a bijection between $J$-holomorphic curves and $J^{c}$-holomorphic curves. So with these choices, the canonical identification of generators is an isomorphism of chain complexes. Moreover, it is shown in [10] that the resulting isomorphism (2.1) does not depend on $J$ (under the canonical isomorphisms between the versions of ECH defined using different almost complex structures).

Theorem 2.4. Let $\left(Y_{+}, \lambda_{+}\right)$and $\left(Y_{-}, \lambda_{-}\right)$be closed oriented 3-manifolds with nondegenerate contact forms. Let $(X, \lambda)$ be an exact symplectic cobordism from $\left(Y_{+}, \lambda_{+}\right)$ to $\left(Y_{-}, \lambda_{-}\right)$. Then there exist maps (of ungraded $\mathbb{Z} / 2$-modules)

$$
\Phi^{L}(X, \lambda): E C H_{*}^{L}\left(Y_{+}, \lambda_{+}\right) \longrightarrow E C H_{*}^{L}\left(Y_{-}, \lambda_{-}\right)
$$

for each real number $L$, such that:

(Homotopy Invariance) The map $\Phi^{L}(X, \lambda)$ depends only on $L$ and the homotopy class of $(X, \omega)$.

(Inclusion) If $L<L^{\prime}$ then the following diagram commutes:

$$
\begin{array}{ccc}
E C H_{*}^{L}\left(Y_{+}, \lambda_{+}\right) \stackrel{\Phi^{L}(X, \lambda)}{\longrightarrow} & \operatorname{ECH}_{*}^{L}\left(Y_{-}, \lambda_{-}\right) \\
\downarrow^{{ }^{L, L^{\prime}}} & & \downarrow^{L, L^{\prime}} \\
\operatorname{ECH}_{*}^{L^{\prime}}\left(Y_{+}, \lambda_{+}\right) \stackrel{\Phi^{L^{\prime}}(X, \lambda)}{\longrightarrow} & E C H_{*}^{L^{\prime}}\left(Y_{-}, \lambda_{-}\right) .
\end{array}
$$

(Direct Limit)

$$
\lim _{L \rightarrow \infty} \Phi^{L}(X, \lambda)=\Phi(X): E C H_{*}\left(Y_{+}, \lambda_{+}\right) \longrightarrow E C H_{*}\left(Y_{-}, \lambda_{-}\right),
$$

where $\Phi(X)$ is as in Definition 1.5.

(Composition) If $(X, \lambda)$ is the composition of $\left(X_{2}, \lambda_{2}\right)$ and $\left(X_{1}, \lambda_{1}\right)$ as above with $\lambda_{0}$ nondegenerate, then

$$
\Phi^{L}\left(X_{2} \circ X_{1}, \lambda\right)=\Phi^{L}\left(X_{2}, \lambda_{2}\right) \circ \Phi^{L}\left(X_{1}, \lambda_{1}\right) .
$$


(Scaling) If c is a positive constant then the following diagram commutes:

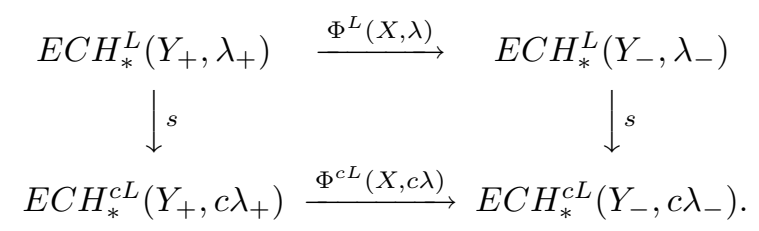

(Holomorphic Curves) Let $J$ be a cobordism-admissible almost complex structure on $\bar{X}$ such that $J_{+}$and $J_{-}$are ECH-generic. Then there exists a (noncanonical) chain map

$$
\hat{\Phi}^{L}: E C C_{*}^{L}\left(Y_{+}, \lambda_{+} ; J_{+}\right) \longrightarrow E C C_{*}^{L}\left(Y_{-}, \lambda_{-} ; J_{-}\right)
$$

inducing $\Phi^{L}(X, \lambda)$, such that if $\Theta^{+}$and $\Theta^{-}$are ECH generators for $\left(Y_{+}, \lambda_{+}\right)$ and $\left(Y_{-}, \lambda_{-}\right)$respectively with action less than $L$, then:

(i) If there are no broken $J$-holomorphic curves in $\bar{X}$ from $\Theta^{+}$to $\Theta^{-}$, then $\left\langle\hat{\Phi}^{L} \Theta^{+}, \Theta^{-}\right\rangle=0$.

(ii) If the only broken J-holomorphic curve in $\bar{X}$ from $\Theta^{+}$to $\Theta^{-}$is a union of covers of product cylinders, then $\left\langle\hat{\Phi}^{L} \Theta^{+}, \Theta^{-}\right\rangle=1$.

Example 2.5. For any three-manifold with a nondegenerate contact form, the empty set of Reeb orbits is a cycle in the ECH chain complex (whose homology class in ECH corresponds to the "contact invariant" in Seiberg-Witten Floer cohomology, see [19]). If $\emptyset_{ \pm}$denotes the empty set of Reeb orbits, regarded as a generator of the ECH chain complex for $Y_{ \pm}$, then it follows from the Holomorphic Curves axiom that

$$
\Phi^{L}(X, \lambda):\left[\emptyset_{+}\right] \longmapsto\left[\emptyset_{-}\right] .
$$

The reason is that for any cobordism-admissible almost complex structure $J$, by (1.9) there is a unique $J$-holomorphic curve with no positive end, namely the empty holomorphic curve.

Remark 2.6. Theorem 2.4 has applications beyond the chord conjecture, for example to symplectic embedding obstructions [7]. The Scaling axiom is not needed for the proof of the chord conjecture, but is useful in these other applications.

\section{Legendrian surgery}

We now explain the details of the Legendrian surgery construction we will use. In particular we define a sequence of Legendrian surgeries, related to each other by exact symplectic cobordisms, in which the Reeb vector field is increasingly well-behaved.

To begin, recall that a Liouville vector field on a symplectic manifold $(X, \omega)$ is a vector field $V$ such that $\mathcal{L}_{V} \omega=\omega$. A Liouville vector field $V$ is equivalent to a 1 -form $\lambda$ such that $d \lambda=\omega$, via the equation $\lambda=\imath_{V} \omega$.

If $Y$ is a hypersurface in $(X, \omega)$ transverse to a Liouville vector field $V$, then $\lambda_{Y}:=\left.\lambda\right|_{Y}$ is a contact form on $Y$. Now let $Y^{\prime}$ be another hypersurface transverse to $V$, and suppose that the time $t$ flow of $V$ defines a diffeomorphism $\phi: Y \rightarrow Y^{\prime}$, where $t$ is some function on $Y$. Then the contact forms on $Y$ and $Y^{\prime}$ are related by

$$
\phi^{*} \lambda_{Y^{\prime}}=e^{t} \lambda_{Y}
$$


With the above preliminaries out of the way, consider now a closed oriented 3manifold $Y_{0}$ with a nondegenerate contact form $\lambda_{0}$. Let $\mathcal{K}$ be a Legendrian knot in $\left(Y_{0}, \lambda_{0}\right)$. Let $Y_{1}$ be the 3 -manifold obtained by surgery along $\mathcal{K}$ with framing $t b(\mathcal{K})-1$.

Proposition 3.1. There exist:

- a nondegenerate contact form $\lambda_{1}$ on $Y_{1}$,

- an exact symplectic cobordism $(X, \lambda)$ from $\left(Y_{1}, \lambda_{1}\right)$ to $\left(Y_{0}, \lambda_{0}\right)$; let $V$ denote its associated associated Liouville vector field;

- a compact hypersurface $Y_{1 / n}$ in $X$ transverse to $V$ for each positive integer $n$,

- and neighborhoods $U_{1 / n}$ of $\mathcal{K}$ in $Y_{0}$ with $U_{1 / n} \supset U_{1 /(n+1)}$ and $\bigcap_{n=1}^{\infty} U_{1 / n}=\mathcal{K}$,

with the following properties:

(a) The induced contact form $\lambda_{1 / n}$ on $Y_{1 / n}$ is nondegenerate.

(b) The negative time flow of $V$ induces a diffeomorphism $Y_{1 / n} \stackrel{\simeq}{\rightarrow} Y_{1 /(n+1)}$. (The flow time varies over $Y_{1 / n}$.)

(c) The time $-1 / n$ flow of $V$, call it $\phi_{-1 / n}$, is defined on all of $Y_{1 / n}$. There is a subset $\widetilde{U}_{1 / n}$ of $Y_{1 / n}$ such that

$$
\phi_{-1 / n}\left(Y_{1 / n} \backslash \widetilde{U}_{1 / n}\right)=Y_{0} \backslash U_{1 / n} .
$$

(d) The Reeb vector field on $\left(Y_{1 / n}, \lambda_{1 / n}\right)$ has no closed orbits contained entirely within $\widetilde{U}_{1 / n}$.

Proof. The idea for building the cobordism $(X, \lambda)$ is to start with the exact symplectic cobordism $\left([0,1] \times Y_{0}, e^{s} \lambda_{0}\right)$, where $s$ denotes the $[0,1]$ coordinate. One then attaches a 2-handle with an appropriate Liouville form to $\{1\} \times Y_{0}$ in a neighborhood of $\{1\} \times \mathcal{K}$. We proceed in four steps.

Step 1. We first describe a model for the handle attachment, following [20]. Consider $\mathbb{R}^{4}$ with coordinates $q_{1}, q_{2}, p_{1}, p_{2}$ and the symplectic form $\omega=\sum_{i=1}^{2} d p_{i} d q_{i}$. Define a Liouville vector field on $\mathbb{R}^{4}$ by

$$
V=\sum_{i=1}^{2}\left(-p_{i} \frac{\partial}{\partial p_{i}}+2 q_{i} \frac{\partial}{\partial q_{i}}\right) .
$$

Consider the hypersurface

$$
Y=\left(p_{1}^{2}+p_{2}^{2}=1\right) \subset \mathbb{R}^{4},
$$

regarded as the boundary of $\left(p_{1}^{2}+p_{2}^{2} \geq 1\right)$. The Liouville vector field $V$ is transverse to $Y$ and so induces a contact form on $Y$. With respect to this contact form, the circle

$$
C=\left(q_{1}=q_{2}=0, p_{1}^{2}+p_{2}^{2}=1\right)
$$

is a Legendrian knot. To the region $\left(p_{1}^{2}+p_{2}^{2} \geq 1\right)$ we now attach the 2-handle consisting of the subset of $\mathbb{R}^{4}$ where

$$
p_{1}^{2}+p_{2}^{2} \leq 1, \quad q_{1}^{2}+q_{2}^{2} \leq \varepsilon
$$

for some $\varepsilon>0$. The boundary of the region with the 2-handle attached has a corner where $p_{1}^{2}+p_{2}^{2}=1$ and $q_{1}^{2}+q_{2}^{2}=\varepsilon$. To round the corner, we replace the boundary 
hypersurface $q_{1}^{2}+q_{2}^{2}=\varepsilon$ of the handle with a nearby hypersurface, staying within the region $\varepsilon / 2<q_{1}^{2}+q_{2}^{2} \leq \varepsilon$, and defined by an equation of the form

$$
f\left(q_{1}^{2}+q_{2}^{2}, p_{1}^{2}+p_{2}^{2}\right)=0
$$

where at each point on the zero set of $f$ we have $\partial f / \partial x>0$ and $\partial f / \partial y \leq 0$. The boundary of the region with the 2-handle attached is then a smooth hypersurface $Y^{\prime}$ which is transverse to the Liouville vector field $V$.

Step 2. We now pass from the model case to the case of interest. By [20, Prop. 4.2], there is a diffeomorphism of a neighborhood $N$ of $C$ in $\mathbb{R}^{4}$ with a neighborhood of $\{1\} \times$ $\mathcal{K}$ in the symplectization $\mathbb{R} \times Y_{0}$, which respects the symplectic forms and Liouville vector fields and locally identifies the hypersurface $Y$ in $\mathbb{R}^{4}$ with the hypersurface $\{1\} \times Y_{0}$ in $\mathbb{R} \times Y_{0}$. If $\varepsilon>0$ is sufficiently small, then $N \cap Y$ will contain the region in $Y$ to which the 2 -handle in $\mathbb{R}^{4}$ is attached. We then use the above diffeomorphism to attach the 2-handle described above in $\mathbb{R}^{4}$, with its symplectic form and Liouville vector field, to $[0,1] \times Y_{0}$. We now provisionally define $X$ to be the resulting exact symplectic cobordism, and $\left(Y_{1}, \lambda_{1}\right)$ to be its positive boundary with the induced contact form. It is not hard to check that as a smooth 3-manifold, $Y_{1}$ is obtained from $Y_{0}$ by surgery on $\mathcal{K}$ with framing $t b(\mathcal{K})-1$. We also define $\widetilde{U}_{1}$ to be the part of $Y_{1}$ in the handle, and $U_{1}=Y_{0} \backslash \phi_{-1}\left(Y_{1} \backslash \widetilde{U}_{1}\right)$. That is, $U_{1} \subset Y_{0}$ corresponds to the subset of $\{1\} \times Y_{0}$ to which the handle is attached.

Step 3. We now check that the Reeb vector field $R_{1}$ on $Y_{1}$ has the required properties. We first show that $R_{1}$ has no closed orbit contained in $\widetilde{U}_{1}$. On $\widetilde{U}_{1}$, in terms of the coordinates on $\mathbb{R}^{4}$, the Reeb vector field $R_{1}$ is parallel to the Hamiltonian vector field associated to the function (3.2). Thus

$$
g R_{1}=\frac{\partial f}{\partial x}\left(q_{1} \frac{\partial}{\partial p_{1}}+q_{2} \frac{\partial}{\partial p_{2}}\right)-\frac{\partial f}{\partial y}\left(p_{1} \frac{\partial}{\partial q_{1}}+p_{2} \frac{\partial}{\partial q_{2}}\right)
$$

where $g$ is some positive function on $\widetilde{U}_{1}$. Now define another function $h$ on $\widetilde{U}_{1}$ by

$$
h:=p_{1} q_{1}+p_{2} q_{2} \text {. }
$$

We then compute that

$$
R_{1}(h)>0
$$

on all of $\widetilde{U}_{1}$. It follows immediately that $R_{1}$ has no closed orbit contained in $\widetilde{U}_{1}$.

Next we consider nondegeneracy of $\lambda_{1}$. By construction, $\lambda_{1}$ is a constant multiple of $\lambda_{0}$ outside of $\widetilde{U}_{1}$. Since $\lambda_{0}$ was assumed nondegenerate, it follows that any Reeb orbit for $\lambda_{1}$ that avoids the region $\widetilde{U}_{1}$ is nondegenerate as well. Consequently we can make $\lambda_{1}$ nondegenerate by perturbing it (specifically, multiplying it by a positive function close to 1 ) in $\widetilde{U}_{1}$. By equation (3.1), such a perturbation of $\lambda_{1}$ can be effected by perturbing the hypersurface $\widetilde{U}_{1}$ in the definition of $X$. If this perturbation is sufficiently $C^{1}$-small, then (3.3) will still hold, so the Reeb vector field of $\lambda_{1}$ will now have all of the required properties.

Step 4. The hypersurface $Y_{1 / n} \subset X$ is now defined to be the positive boundary of the region obtained by starting with $[0,1 / n] \times Y_{0}$ and attaching a taller and thinner 2 -handle. This handle is obtained by starting with the subset of $\mathbb{R}^{4}$ where

$$
p_{1}^{2}+p_{2}^{2} \leq e^{2(1-1 / n)}, \quad q_{1}^{2}+q_{1}^{2} \leq 2^{1-n} \varepsilon,
$$


then rounding corners as before and perturbing if necessary to make $\lambda_{1 / n}$ nondegenerate. Finally, one defines $\widetilde{U}_{1 / n}$ to be the part of $Y_{1 / n}$ in the handle, and $U_{1 / n}=Y_{0} \backslash \phi_{-1 / n}\left(Y_{1} \backslash \widetilde{U}_{1}\right)$.

A basic consequence of the above construction is the following:

Lemma 3.2. Suppose $\mathcal{K}$ has no Reeb chord with action $\leq L$. Then for all $n$ sufficiently large:

(a) The Reeb orbits of $\lambda_{1 / n}$ with action $<e^{1 / n} L$ avoid the region $\widetilde{U}_{1 / n}$.

(b) $\phi_{-1 / n}$ defines a bijection from the Reeb orbits of $\lambda_{1 / n}$ with action $<e^{1 / n} L$ to the Reeb orbits of $\lambda_{0}$ with action $<L$.

Proof. Suppose $\left\{n_{k}\right\}_{k=1,2, \ldots}$ is an increasing sequence of positive integers and that for each $k$ there exists a Reeb orbit $\gamma_{k}$ for $\lambda_{1 / n_{k}}$ of action $<e^{1 / n_{k}} L$ intersecting $\widetilde{U}_{1 / n_{k}}$. Then for each $k$, the set $\phi_{-1 / n_{k}}\left(\gamma_{k} \cap\left(Y_{1 / n_{k}} \backslash \widetilde{U}_{1 / n_{k}}\right)\right)$ is a union of Reeb trajectories of $\lambda_{0}$ starting and ending on the boundary of $U_{1 / n_{k}}$ with total action less than $L$. Picking one of these trajectories for each $k$, we can pass to a subsequence so that these trajectories converge to a Reeb chord with action $\leq L$. This proves (a). Similarly, if $n$ is sufficiently large then the Reeb orbits of $\lambda_{0}$ with action $<L$ avoid the region $U_{1 / n}$. This together with (a) implies (b).

\section{The chord conjecture: nondegenerate case}

We now prove the chord conjecture, Theorem 1.1, in the case when the contact form $\lambda_{0}$ is nondegenerate. Below, we use the notation from the Legendrian surgery construction in Proposition 3.1. Also, to shorten the notation we write $H_{*}(Y, \lambda)$ to denote $E C H_{*}(Y, \lambda)$, and $H_{*}^{L}(Y, \lambda)$ to denote $E C H_{*}^{L}(Y, \lambda)$.

Observe that by the construction in $\S 3$, the exact symplectic cobordism $(X, \lambda)$ contains an exact symplectic cobordism from $\left(Y_{1 / n}, \lambda_{1 / n}\right)$ to $\left(Y_{0}, \lambda_{0}\right)$, call this $X_{1 / n}$. The main lemma is now:

Lemma 4.1. Let $L>0$. Suppose that $\mathcal{K}$ has no Reeb chord of action $\leq L$. Then for all $n$ sufficiently large, the cobordism map

$$
\Phi^{e^{1 / n} L}\left(X_{1 / n}, \lambda\right): H_{*}^{e^{1 / n} L}\left(Y_{1 / n}, \lambda_{1 / n}\right) \longrightarrow H_{*}^{e^{1 / n} L}\left(Y_{0}, \lambda_{0}\right)
$$

is the composition of an isomorphism

$$
H_{*}^{e^{1 / n} L}\left(Y_{1 / n}, \lambda_{1 / n}\right) \stackrel{\simeq}{\longrightarrow} H_{*}^{L}\left(Y_{0}, \lambda_{0}\right)
$$

with the inclusion-induced map $\imath^{L, e^{1 / n} L}: H_{*}^{L}\left(Y_{0}, \lambda_{0}\right) \rightarrow H_{*}^{e^{1 / n} L}\left(Y_{0}, \lambda_{0}\right)$.

Proof. By Lemma 3.2, if $n$ is sufficiently large, then the Reeb orbits for $\lambda_{1 / n}$ of action less than $e^{1 / n} L$ correspond via $\phi_{-1 / n}$ to the Reeb orbits for $\lambda_{0}$ of action less than $L$, and the latter stay outside of the neighborhood $U_{1 / n}$ of $\mathcal{K}$. Let $n$ be so large.

By Proposition 3.1(c), the flow of the Liouville vector field $V$ starting on $Y_{1 / n}$ for times in the interval $[-1 / n, 0]$ defines an embedding of $[-1 / n, 0] \times Y_{1 / n}$ into $X_{1 / n}$. Let $X_{1 / n}^{0}$ denote the image of this embedding. We identify $X_{1 / n}^{0}$ with $[0,1 / n] \times Y_{1 / n}$ such that $Y_{1 / n}$ is identified with $\{1 / n\} \times Y_{1 / n}$, and the Liouville vector field $V=\partial / \partial s$, 
where $s$ denotes the $[0,1 / n]$ coordinate. Then $\{0\} \times Y_{1 / n}$ defines a hypersurface in $X_{1 / n}$ which includes $Y_{0} \backslash U_{1 / n} \subset \partial X_{1 / n}$, and which also passes into the interior of $X_{1 / n}$. Let $X_{1 / n}^{1}$ denote $X_{1 / n} \backslash X_{1 / n}^{0}$. We can now decompose the completed cobordism $\overline{X_{1 / n}}$ as

$$
\overline{X_{1 / n}}=\left((-\infty, 0] \times Y_{0}\right) \cup X_{1 / n}^{1} \cup\left([0, \infty) \times Y_{1 / n}\right),
$$

where $X_{1 / n}^{0}$ corresponds to $[0,1 / n] \times Y_{1 / n}$ in (4.3).

We now choose a cobordism-admissible almost complex structure $J$ on $\overline{X_{1 / n}}$ in four steps as follows. First, let $J_{+}$be an almost complex structure on $\mathbb{R} \times Y_{1 / n}$ which is symplectization-admissible with respect to $\lambda_{1 / n}$ and ECH-generic. Require $J$ to agree with $J_{+}$on $[1 / n, \infty) \times Y_{1 / n}$. Second, extend $J$ over $[0,1 / n] \times Y_{1 / n}$ by setting $J=J_{+}$on $\operatorname{Ker}\left(\lambda_{1 / n}\right)$, and $J\left(\partial_{s}\right)=f(s) R_{1 / n}$, where $R_{1 / n}$ denotes the Reeb vector field associated to $\lambda_{1 / n}$, and $f:[0,1 / n] \rightarrow \mathbb{R}$ is a positive function which equals 1 near $s=1 / n$ and which equals $e^{1 / n}$ near $s=0$. Third, extend $J$ over $(-\infty, 0] \times Y_{0}$ so that it agrees with an almost complex structure $J_{-}$on $\mathbb{R} \times Y_{0}$ which is symplectization-admissible for $\lambda_{0}$ and ECH-generic. Note that one can arrange for $J_{-}$to be ECH-generic without disturbing the previous choices because $J_{+}$is ECH-generic. To complete the construction of $J$, choose an arbitrary $\omega$-compatible extension of $J$ over $X_{1 / n}^{1}$.

With the above choices, $[0,1 / n] \times\left(Y_{1 / n} \backslash \widetilde{U}_{1 / n}\right)$ is a product region in the sense of Definition 2.2. In particular, let $\Theta$ be an ECH generator for $\lambda_{1 / n}$ of action less than $e^{1 / n} L$. Since the Reeb orbits in $\Theta$ stay out of the region $\widetilde{U}_{1 / n}$, there is a union of covers of product cylinders (see Definition 2.3) in $\overline{X_{1 / n}}$ from $\Theta$ to $\phi_{-1 / n}(\Theta)$.

We claim that if $C$ is any other $J$-holomorphic curve in $\overline{X_{1 / n}}$ from the above $\Theta$ to an ECH generator $\Theta^{\prime}$ for $\lambda_{0}$, then

$$
e^{-1 / n} \mathcal{A}(\Theta)>\mathcal{A}\left(\Theta^{\prime}\right),
$$

where $\mathcal{A}$ denotes the symplectic action. To prove (4.4), observe that the Liouville form $\lambda$ on $\overline{X_{1 / n}}$ agrees with $e^{s} \lambda_{0}$ on $(-\infty, 0] \times Y_{0}$ and agrees with $e^{s-1 / n} \lambda_{1 / n}$ on $[0, \infty) \times Y_{1 / n}$ in $(4.3)$. Using Stokes' theorem, we obtain

$$
\begin{aligned}
e^{-1 / n} \mathcal{A}(\Theta)-\mathcal{A}\left(\Theta^{\prime}\right)= & \int_{C \cap\left([0, \infty) \times Y_{1 / n}\right)} d\left(e^{-1 / n} \lambda_{1 / n}\right) \\
& +\int_{C \cap X_{1 / n}^{1}} d \lambda \\
& +\int_{C \cap\left((-\infty, 0] \times Y_{0}\right)} d \lambda_{0} .
\end{aligned}
$$

The first and third integrals on the right are pointwise nonnegative, and zero only where $C$ is tangent to $\partial_{s}$. The second integral on the right is pointwise positive. We conclude that $e^{-1 / n} \mathcal{A}(\Theta)-\mathcal{A}\left(\Theta^{\prime}\right) \geq 0$, with equality if and only if $C$ is a union of covers of product cylinders.

Consider now the chain map $\hat{\Phi}^{e^{1 / n} L}\left(X_{1 / n}, \lambda\right)$ inducing (4.1) provided by the Holomorphic Curves axiom in Theorem 2.4. It follows from (4.4) that this chain map is a 
composition of chain maps

$$
E C C_{*}^{e^{1 / n} L}\left(Y_{1 / n}, \lambda_{1 / n} ; J_{+}\right) \longrightarrow E C C_{*}^{L}\left(Y_{0}, \lambda_{0} ; J_{-}\right) \longrightarrow E C C_{*}^{e^{1 / n} L}\left(Y_{0}, \lambda_{0} ; J_{-}\right),
$$

where the map on the right is the inclusion, and the map on the left is triangular with respect to the identification of generators induced by $\phi_{-1 / n}$. In particular the left map is an isomorphism of chain complexes, and hence induces an isomorphism on homology.

To proceed, we now define quantitative measures of the failure of the cobordism map (1.10) to be an isomorphism.

Definition 4.2. $\quad$ (a) Define $A$ to be the infimum of the set of real numbers $L$ such that the image of the inclusion-induced map

$$
\imath^{L}: H_{*}^{L}\left(Y_{0}, \lambda_{0}\right) \longrightarrow H_{*}\left(Y_{0}, \lambda_{0}\right)
$$

is not contained in the image of the cobordism map (1.10).

(b) Define $B$ to be the infimum of the set of real numbers $L$ such that the kernel of the cobordism map

$$
\Phi^{L}(X, \lambda): H_{*}^{L}\left(Y_{1}, \lambda_{1}\right) \longrightarrow H_{*}^{L}\left(Y_{0}, \lambda_{0}\right)
$$

is not contained in the kernel of the inclusion-induced map

$$
\imath^{L}: H_{*}^{L}\left(Y_{1}, \lambda_{1}\right) \longrightarrow H_{*}\left(Y_{1}, \lambda_{1}\right) \text {. }
$$

Lemma 4.3. (a) If (1.10) is not surjective, then $A<\infty$.

(b) If (1.10) is not injective, then $B<\infty$.

Proof. (a) If (1.10) is not surjective then there exists an element of $H_{*}\left(Y_{0}, \lambda_{0}\right)$ which is not in the image; and by (1.4), any given element of $H_{*}\left(Y_{0}, \lambda_{0}\right)$ comes from $H_{*}^{L}\left(Y_{0}, \lambda_{0}\right)$ for some $L$.

(b) If (1.10) is not injective, then there exists a nonzero element $H_{*}\left(Y_{1}, \lambda_{1}\right)$ which maps to zero in $H_{*}\left(Y_{0}, \lambda_{0}\right)$. We can represent the former by a chain $\zeta$ of action less than some $L$, and its image under the cobordism chain map is the boundary of a chain with action less than some $L^{\prime}$. We may assume that $L^{\prime} \geq L$. It then follows from the Inclusion axiom in Theorem 2.4 that $\imath^{L, L^{\prime}}[\zeta]$ is in the kernel of the cobordism map $\Phi^{L^{\prime}}(X, \lambda)$. But $\imath^{L, L^{\prime}}[\zeta]$ is not in the kernel of the inclusion-induced map $\imath^{L^{\prime}}$, because $\imath^{L^{\prime}} \imath^{L, L^{\prime}}[\zeta]=\imath^{L}[\zeta] \neq 0$ in $H_{*}\left(Y_{1}, \lambda_{1}\right)$. Thus $B \leq L^{\prime}$.

In view of Lemma 1.7 and Definition 1.5, the chord conjecture in the nondegenerate case now follows from:

Theorem 4.4. Let $Y_{0}$ be a closed oriented 3-manifold with a nondegenerate contact form $\lambda_{0}$, and let $\mathcal{K}$ be a Legendrian knot in $\left(Y_{0}, \lambda_{0}\right)$.

(a) If (1.10) is not surjective, then $\mathcal{K}$ has a Reeb chord of action $\leq A$.

(b) If (1.10) is not injective, then $\mathcal{K}$ has a Reeb chord of action $\leq B$. 
Proof. To prove part (a), it is enough to show that given $L>0$, if there is no Reeb chord of action $\leq L$, then $A \geq L$. (Because then if $A$ is finite, then there exists a Reeb chord of action $\leq A+1 / n$ for every positive integer $n$, so a compactness argument shows that there exists a Reeb chord of action $\leq A$.) To show that $A \geq L$, it is enough to show that the image of the inclusion-induced map

$$
\imath^{L}: H_{*}^{L}\left(Y_{0}, \lambda_{0}\right) \longrightarrow H_{*}\left(Y_{0}, \lambda_{0}\right)
$$

is contained in the image of the cobordism map (1.10). By the Inclusion and Direct Limit axioms in Theorem 2.4 we have a commutative diagram

$$
\begin{aligned}
& H_{*}^{e^{1 / n} L}\left(Y_{1 / n}, \lambda_{1 / n}\right) \stackrel{\Phi^{e^{1 / n} L}\left(X_{1 / n}, \lambda\right)}{\longrightarrow} H_{*}^{e^{1 / n} L}\left(Y_{0}, \lambda_{0}\right) \\
& \downarrow i^{e^{1 / n} L} \quad \downarrow \downarrow e^{e^{1 / n_{L}}} \\
& H_{*}\left(Y_{1 / n}, \lambda_{1 / n}\right) \stackrel{\Phi\left(X_{1 / n}\right)}{\longrightarrow} \quad H_{*}\left(Y_{0}, \lambda_{0}\right)
\end{aligned}
$$

If $n$ is sufficiently large as in Lemma 4.1, then it follows that we have a commutative diagram

$$
\begin{array}{rlll} 
& H_{*}^{e^{1 / n} L}\left(Y_{1 / n}, \lambda_{1 / n}\right) & \simeq & H_{*}^{L}\left(Y_{0}, \lambda_{0}\right) \\
& \downarrow_{\imath^{e^{1 / n} L}} & & \\
H_{*}\left(Y_{1}, \lambda_{1}\right) \stackrel{\imath^{L}}{\longrightarrow} H_{*}\left(Y_{1 / n}, \lambda_{1 / n}\right) & \stackrel{\Phi\left(X_{1 / n}\right)}{\longrightarrow} H_{*}\left(Y_{0}, \lambda_{0}\right) .
\end{array}
$$

Here the lower left arrow is induced by the cobordism $\overline{X \backslash X_{1 / n}}$ from $Y_{1}$ to $Y_{1 / n}$; this map is an isomorphism because the cobordism $\overline{X \backslash X_{1 / n}}$ is diffeomorphic to the product $[0,1] \times Y_{1}$, and product cobordisms induce isomorphisms on SeibergWitten Floer cohomology. In addition, the composition of the lower two arrows is the cobordism map (1.10), by the Composition axiom in Theorem 2.4 (or by the composition property for $\widehat{H M}^{*}$ ). The statement we need to prove now follows by chasing the diagram.

The proof of part (b) is similar to the proof of part (a). It is enough to show that if there is no Reeb chord of action $\leq L$, then the kernel of the cobordism map $H_{*}^{L}\left(Y_{1}, \lambda_{1}\right) \rightarrow H_{*}^{L}\left(Y_{0}, \lambda_{0}\right)$ is contained in the kernel of the inclusion-induced map $H_{*}^{L}\left(Y_{1}, \lambda_{1}\right) \rightarrow H_{*}\left(Y_{1}, \lambda_{1}\right)$. To do so, let $n$ be sufficiently large as in Lemma 4.1 (with $L$ replaced by $e^{-1 / n} L$ ). Then by the Inclusion and Direct Limit axioms in Theorem 2.4, we have a commutative diagram

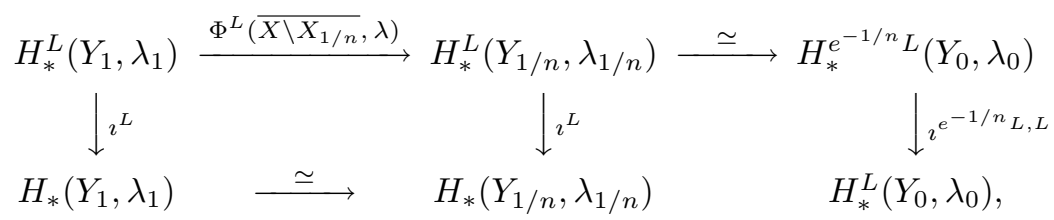

where the composition of the two rightmost arrows is $\Phi^{L}\left(X_{1 / n}, \lambda\right)$. By the Composition axiom in Theorem 2.4, the composition of the three arrows from $H_{*}^{L}\left(Y_{1}, \lambda_{1}\right)$ to $H_{*}^{L}\left(Y_{0}, \lambda_{0}\right)$ is $\Phi^{L}(X, \lambda)$. Now suppose $\zeta \in H_{*}^{L}\left(Y_{1}, \lambda_{1}\right)$ maps to zero in $H_{*}^{L}\left(Y_{0}, \lambda_{0}\right)$. Since the latter is the direct limit of $H_{*}^{e^{-1 / n} L}\left(Y_{0}, \lambda_{0}\right)$ as $n \rightarrow \infty$, it follows that if $n$ is 
chosen sufficiently large then $\zeta$ maps to zero in $H_{*}^{e^{-1 / n} L}\left(Y_{0}, \lambda_{0}\right)$. Chasing the diagram then shows that $\zeta$ maps to zero in $H_{*}\left(Y_{1}, \lambda_{1}\right)$, as required.

\section{The chord conjecture: degenerate case}

We now use Theorem 4.4 for the nondegenerate case to deduce the chord conjecture when $\lambda_{0}$ is degenerate.

When $\lambda_{0}$ is degenerate, one can repeat the surgery construction from $\S 3$, to obtain an exact symplectic cobordism $(X, \lambda)$ as before, now with degenerate contact forms $\lambda_{1 / n}$ on the hypersurfaces $Y_{1 / n}$. However by (3.1) one can perturb these hypersurfaces slightly, as well as the boundary hypersurfaces $Y_{0}$ and $Y_{1}$, in the completion $\bar{X}$, so as to make the induced contact forms on them nondegenerate. In particular, for each positive integer $k$ we can find functions $f_{1 / n}^{(k)}$ on $Y_{1 / n}$ and $f_{0}^{(k)}$ on $Y_{0}$ with

$$
\left\|f_{1 / n}^{(k)}\right\|_{C^{1}},\left\|f_{0}^{(k)}\right\|_{C^{1}}<1 / k
$$

such that we have an exact cobordism $X^{(k)}$ as in $\S 3$, contained in $\bar{X}$ with the same Liouville form $\lambda$ (which we henceforth omit from the notation), with nondegenerate contact forms $\lambda_{1 / n}^{(k)}=e^{f_{1 / n}^{(k)}} \lambda_{1 / n}$ on $Y_{1 / n}$ and $\lambda_{0}^{(k)}=e^{f_{0}^{(k)}} \lambda_{0}$ on $Y_{0}$. We can also assume that

$$
f_{0}^{(1)}>f_{0}^{(k)}, \quad f_{1}^{(1)}>f_{1}^{(k)},
$$

which will be convenient below.

Now let $A(k)$ and $B(k)$ denote the upper bounds on the action of a Reeb chord coming from the cobordism $X^{(k)}$ from $\left(Y_{1}, \lambda_{1}^{(k)}\right)$ to $\left(Y_{0}, \lambda_{0}^{(k)}\right)$. By Theorem 4.4 and a compactness argument for Reeb chords explained at the end of this section, to prove the chord conjecture for $\lambda_{0}$ it is enough to show that $A(k)$ and $B(k)$ stay bounded as $k \rightarrow \infty$. In fact we have:

Proposition 5.1. $A(k) \leq A(1)$ and $B(k) \leq B(1)$.

Proof. By the first part of (5.1), there is a subset $X_{+}^{(k)}$ of $\bar{X}$, diffeomorphic to [0,1] $\times Y_{1}$, which defines an exact symplectic cobordism from $\left(Y_{1}, \lambda_{1}^{(1)}\right)$ to $\left(Y_{1}, \lambda_{1}^{(k)}\right)$. Likewise, by the second part of (5.1), there is a subset $X_{-}^{(k)}$ of $\bar{X}$, diffeomorphic to $[0,1] \times Y_{0}$, which is an exact symplectic cobordism from $\left(Y_{0}, \lambda_{0}^{(1)}\right)$ to $\left(Y_{0}, \lambda_{0}^{(k)}\right)$. Let $X_{0}^{(k)}$ denote compact subset of $\bar{X}$ bounded by the negative boundary of $X_{+}^{(k)}$ and the positive boundary of $X_{-}^{(k)}$. This is an exact symplectic cobordism from $\left(Y_{1}, \lambda_{1}^{(k)}\right)$ to $\left(Y_{0}, \lambda_{0}^{(1)}\right)$, and we have the compositions $X_{0}^{(k)} \circ X_{+}^{(k)}=X^{(1)}$ and $X_{-}^{(k)} \circ X_{0}^{(k)}=X^{(k)}$.

Now fix $L$ and consider the diagram

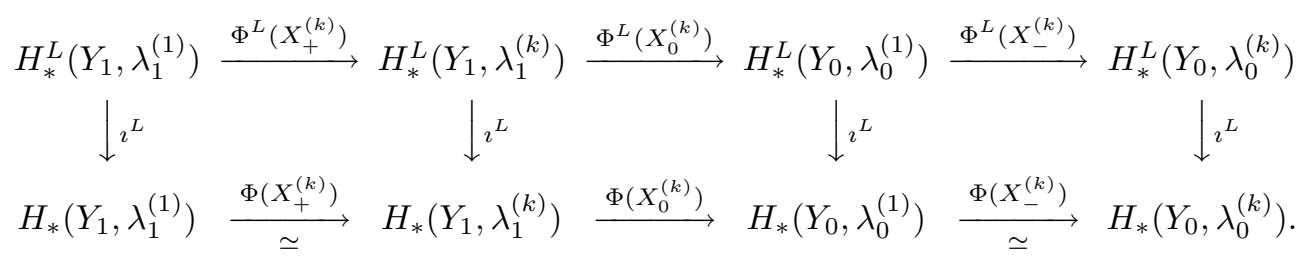


This diagram commutes by the Inclusion axiom, and the maps $\Phi\left(X_{ \pm}^{(k)}\right)$ are isomorphisms because the cobordisms $X_{ \pm}^{(k)}$ are diffeomorphic to products, which induce isomorphisms on Seiberg-Witten Floer cohomology. By the Composition axiom, the composition of the two upper left horizontal arrows is $\Phi^{L}\left(X^{(1)}\right)$, the composition of the two upper right horizontal arrows is $\Phi^{L}\left(X^{(k)}\right)$, the composition of the two lower left horizontal arrows is $\Phi\left(X^{(1)}\right)$, and the composition of the two lower right horizontal arrows is $\Phi\left(X^{(k)}\right)$.

To prove that $A(k) \leq A(1)$, it is enough to show that if the image of $\imath^{L}$ : $H_{*}^{L}\left(Y_{0}, \lambda_{0}^{(k)}\right) \rightarrow H_{*}\left(Y_{0}, \lambda_{0}^{(k)}\right)$ is contained in the image of $\Phi\left(X^{(k)}\right)$, then the image of $\imath^{L}: H_{*}^{L}\left(Y_{0}, \lambda_{0}^{(1)}\right) \rightarrow H_{*}\left(Y_{0}, \lambda_{0}^{(1)}\right)$ is contained in the image of $\Phi\left(X^{(1)}\right)$. This follows immediately by chasing the above diagram.

To prove that $B(k) \leq B(1)$, it is enough to show that if the kernel of $\Phi^{L}\left(X^{(k)}\right)$ is contained in the kernel of $\imath^{L}: H_{*}^{L}\left(Y_{1}, \lambda_{1}^{(k)}\right) \rightarrow H_{*}\left(Y_{1}, \lambda_{1}^{(k)}\right)$, then the kernel of $\Phi^{L}\left(X^{(1)}\right)$ is contained in the kernel of $\imath^{L}: H_{*}^{L}\left(Y_{1}, \lambda_{1}^{(1)}\right) \rightarrow H_{*}\left(Y_{1}, \lambda_{1}^{(1)}\right)$. This also follows immediately from the above diagram.

To conclude, we have:

Proof of Theorem 1.1. Let $Y_{0}$ be a closed oriented 3-manifold with a contact form $\lambda_{0}$, and let $\mathcal{K}$ be a Legendrian knot in $\left(Y_{0}, \lambda_{0}\right)$.

If $\lambda_{0}$ is nondegenerate, then it follows from Lemma 1.7 and Definition 1.5 that the map (1.10) is not an isomorphism. Let $A, B \in[0, \infty]$ be the numbers in Definition 4.2. By Lemma 4.3 we have $\min (A, B)<\infty$, and by Theorem 4.4 the knot $\mathcal{K}$ has a Reeb chord of length at most $\min (A, B)$.

If $\lambda_{0}$ is degenerate, let $\left\{\lambda_{0}^{(k)}\right\}_{k=1,2, \ldots}$ be a sequence of nondegenerate perturbations of $\lambda_{0}$ as described at the beginning of this section, and let $A(k), B(k)$ denote the corresponding quantities from Definition 4.2. By the nondegenerate case, $\mathcal{K}$ has a Reeb chord $\gamma_{k}$ for $\lambda_{0}^{(k)}$ of length at most $\min (A(k), B(k))<\infty$. By Proposition 5.1, the length of $\gamma_{k}$ has a $k$-independent upper bound. Thus we can pass to a subsequence such that the lengths of the Reeb chords $\gamma_{k}$ converge to a real number $L$. We can also pass to a subsequence so that the starting and ending points of the Reeb chords $\gamma_{k}$ converge to points $y_{0}, y_{L} \in \mathcal{K}$. Now as $k \rightarrow \infty$, the 1 -form $\lambda_{0}^{(k)}$ converges to $\lambda_{0}$ in $C^{1}$, and so the Reeb vector field for $\lambda_{0}^{(k)}$ converges to the Reeb vector field for $\lambda_{0}$ in $C^{0}$. Consequently there is a Reeb chord for $\lambda_{0}$ of length $L$ from $y_{0}$ to $y_{L}$.

\section{Acknowledgments}

We thank Jonathan Bloom, Tobias Ekholm, Yasha Eliashberg, Ko Honda, Dusa McDuff, Tomasz Mrowka, and Ivan Smith for helpful discussions. The first author was partially supported by NSF grant DMS-0806037. The second author was partially supported by the Clay Mathematics Insitute, the Mathematical Sciences Research Institute, and the NSF. Both authors thank MSRI, where this work was carried out, for its hospitality. 


\section{References}

[1] C. Abbas, The chord problem and a new method of filling by pseudoholomorphic curves, Int. Math. Res. Not. 2004, no. 18, 913-927.

[2] V. I. Arnold, The first steps of symplectic topology, Russian Math. Surveys 41 (1986), no. 6, $1-21$.

[3] J. Bloom, A link surgery spectral sequence in monopole Floer homology, arXiv:0909.0816.

[4] F. Bourgeois, T. Ekholm, and Y. Eliashberg, Effect of Legendrian surgery, arXiv:0911.0026.

[5] M. Hutchings, The embedded contact homology index revisited, New perspectives and challenges in symplectic field theory, 263-297, CRM Proc. Lecture Notes 49, Amer. Math. Soc., 2009.

[6] M. Hutchings, Embedded contact homology and its applications, in Proceedings of the 2010 ICM.

[7] M. Hutchings, Quantitative embedded contact homology, arXiv:1005.2260, to appear in J. Diff. Geom.

[8] M. Hutchings and C. H. Taubes, Gluing pseudoholomorphic curves along branched covered cylinders I, J. Symplectic Geom. 5 (2007), 43-137.

[9] M. Hutchings and C. H. Taubes, Gluing pseudoholomorphic curves along branched covered cylinders II, J. Symplectic Geom. 7 (2009), 29-133.

[10] M. Hutchings and C. H. Taubes, Proof of the Arnold chord conjecture in three dimensions II, in preparation.

[11] K. Mohnke, Holomorphic disks and the chord conjecture, Ann. of Math. 154 (2001), no. 1, 219-222.

[12] P.B. Kronheimer and T.S. Mrowka, Monopoles and three-manifolds, Cambridge University Press, 2008.

[13] P. Kronheimer, T. Mrowka, P. Ozsváth and Z. Szabó, Monopoles and lens space surgeries, Annals of Math. 165 (2007), 457-546.

[14] J. Latschev and C. Wendl, Algebraic torsion in contact manifolds, arXiv:1009.3262.

[15] C. H. Taubes, Embedded contact homology and Seiberg-Witten Floer homology I, Geometry and Topology 14 (2010), 2497-2581.

[16] C. H. Taubes, Embedded contact homology and Seiberg-Witten Floer homology II, Geometry and Topology 14 (2010), 2583-2720.

[17] C. H. Taubes, Embedded contact homology and Seiberg-Witten Floer homology III, Geometry and Topology 14 (2010), 2721-2817.

[18] C. H. Taubes, Embedded contact homology and Seiberg-Witten Floer homology IV, Geometry and Topology 14 (2010), 2819-2960.

[19] C. H. Taubes, Embedded contact homology and Seiberg-Witten Floer homology V, Geometry and Topology 14 (2010), 2961-3000.

[20] A. Weinstein, Contact surgery and symplectic handlebodies, Hokkaido Math. J. 20 (1991), 241251.

Department of Mathematics, University of California, Berkeley, Berkeley CA 94720

E-mail address: hutching@math.berkeley.edu

Mathematics Department, Harvard University, Cambridge MA 02138

E-mail address: chtaubes@math.harvard.edu 